\title{
Clasificación internacional de enfermedades vs clasificación del funcionamiento, la discapacidad y el estado de salud: la contractura muscular. Reporte de caso.
}

\section{International classification of diseases vs functional classification, disability and health status: muscle contract. Case report.}

Paola Vernaza Pinzón ${ }^{1}$, Jhon Alejandro Sánchez ${ }^{2}$, Carolina Jojoa Cifuentes², María Camila Martínez², Luis David Patińo ${ }^{2}$, Eileen Yulieth Moriones ${ }^{2}$

\section{RESUMEN}

Sepresenta el caso de una paciente remitida por contractura muscular a nivel del músculo trapecio superior izquierdo. La contractura muscular ha sido definida como una contracción permanente, dolorosa e involuntaria de un músculo que no cede con el reposo y su duración oscila

\begin{abstract}
We present a case of a patient referred due to a muscular contracture at the left upper trapezius muscle level, the muscular contracture has been defined as a permanent, painful and involuntary contraction of a muscle, that is not stopping with rest and its duration ranges from five
\end{abstract}

\section{Historial del artículo}

Fecha de recepción: 05/04/2019

Fecha de aprobación: 10/04/2020

1 Universidad del Cauca. Fisioterapeuta. Magister en Dolor en la práctica clínica. Docente Práctica clínica en osteomuscular, I nivel patología crónica. Departamento de Fisioterapia. Popayán, Colombia.

2 Universidad del Cauca, Estudiantes IX semestre Programa de Fisioterapia, práctica clínica en osteomuscular, I nivel patología crónica. Popayán, Colombia.

Correspondencia: Paola Vernaza Pinzón. Dirección: Carrera 6 Nº 13N-50 sector La Estancia, Popayán, Cauca. Teléfono: 8209870 8209800 ext. 2717. Correo electrónico: pvernaza@unicauca.edu.co

Como citar este artículo: Vernaza-Pinzón P, Sánchez JA, Jojoa-Cifuentes C, Martínez MC, Patińo LD, Moriones E. Clasificación internacional de enfermedades vs clasificación del funcionamiento, la discapacidad y el estado de salud: : la contractura muscular. Reporte de caso. propósito de un caso. Revista de la Facultad de Ciencias de la Salud de la Universidad del Cauca. 2020; 22 (1): 51 - 59 
entre cinco y diez días. Es el médico quien realiza, a partir de una valoración minuciosa, el diagnóstico basado en la CIE-11 donde considera la remisión del paciente a diferentesprofesionales entre ellos el fisioterapeuta, quien se encarga de realizar una valoración en torno al estado de salud de estructuras, funciones, limitación de la actividad, restricción en la participación, factores ambientales y factorespersonales. A pesar que el fisioterapeuta no emite un diagnóstico basado en la CIE-11, sídetermina el estado de funcionamiento, discapacidad y el estado de salud de una persona con el fin de establecer un plan de tratamiento desde una perspectiva de bienestary calidad de vida, superando la mirada biomédica al incorporar una mirada biopsicosocial al funcionamiento y la discapacidad humana.

Palabras Clave: Clasificación internacional de enfermedades, CIE-10, Clasificación internacional del funcionamiento, De la discapacidad y de la salud, Contractura, Reporte de caso.

\section{INTRODUCCIÓN}

La Clasificación Internacional de Enfermedades (CIE), es fundamental para la comunicación sobre los problemas de salud. Desde la versión anterior (CIE-10), se incluía una amplia gama de problemas relacionados con la salud y no solo la enfermedad en el sentido estricto. Con su modelo multiaxial, la CIE ofrece al profesional ofrece al profesional de la medicina aproximarse a la condición total del paciente, diagnosticando todos sus trastornos, así como el impacto de éstos sobre el funcionamiento del individuo y el contexto en el cual vive (1).

Entre tanto, el modelo de la clasificación internacional del funcionamiento, de la discapacidad y de la salud (CIF), constituye el marco conceptual de la OMS para describir la salud y los aspectos relacionados, dado que se basa en dos principios: 1. La universalidad, el funcionamiento/ discapacidad que son inmanentes a la condición humana y 2. La continuidad, persiste a lo largo de toda la vida (2). Las clasificaciones CIE y la CIF, son complementarias, brindan información sobre el diagnóstico, unida a la del funcionamiento con una visión más amplia sobre el estado de salud de las personas (3).

El dolor musculoesquelético es un problema de salud que afecta hasta el $80 \%$ de la población mundial; de este porcentaje un $10 \%$ a $20 \%$ se clasifica como dolor crónico (5). El dolor se manifiesta por una amplificación excesiva de la seńal dolorosa dada por el sistema nervioso central to ten days. It's the doctor who makes the diagnosis of the CIE-11 from a thorough assessment, considering the referral of patient to different professionals including the physiotherapist, who is responsible for making an assessment about the health status of structures, functions, limitation of activity, restriction of participation, environmental factors and personal factors. Although the physiotherapistdoesn't issue a diagnosis based on the CIE-11, he determines the working status, disability and health status of a person, in orden to establish a treatment plan from a well-being and quality oflife perspective, overcoming the biomedical perspective incorporating a biopsychosocial perspective to the human functioning and disability.

Key words: International classification of diseases, CIE-10, International classification of functioning, Disability and health, Contracture, Case reports.

y el sistema nervioso periférico; adicionalmente, puede existir susceptibilidad genética que se active por un estímulo ambiental incluso el estrés. Es entonces como esta situación de salud genera deterioro funcional y de la calidad de vida de las personas (6), entendiendo que si se pierde la movilidad, se afecta la calidad de vida.

Como causa importante del dolor musculoesquelético crónico deben considerarse alteraciones de las partes blandas como los espasmos musculares que se analizan como una contractura (7). Esta se define como una contracción involuntaria, duradera o permanente de uno o varios grupos musculares. A la palpación, el musculo afectado tiene hipersensibilidad que aumenta cuando el paciente realiza una contracción muscular contra resistencia indicando una elasticidad muscular reducida (8).

El músculo está expuesto a recibir micro traumas a repetición; en aquellos puntos donde se reciben traumas, la fibra muscular puede lesionarse y contracturarse, aumentando la tensión y el tono lo que la convierte en una zona dolorosa llamada punto gatillo (9). Este desorden no inflamatorio de origen musculoesquelético, asociado con dolor y rigidez muscular, se manifiesta como un foco hiperirritable en una banda tensa de un músculo esquelético, que puede provocar disfunción muscular, dolor referido y otros signos y síntomas como parestesias, disestesias, visión borrosa, sudoración, lagrimeo, enrojecimiento cutáneo y cambios vasomotores y térmicos (10). Entonces, la contractura deja de ser contractura para pasar a constituirse en un síndrome de dolor miofascial 
caracterizado por una etiología multifactorial, afectando más a las mujeres entre 20 y 40 ańos en una relación de 3:1 a 5:1 respecto a los hombres (9).

Ahora bien, existe un síndrome denominado síndrome de sensibilización central, definido como una amplificación de la seńal neural dentro del sistema nervioso central que desencadena hipersensibilidad al dolor; este síndrome ha sido recientemente reconocido como importante en la fisiopatología del dolor crónico, trastornos de la articulación temporomandibular, dolor lumbar crónico, latigazo cervical y síndrome de dolor miofascial, en los que el dolor es persistente sin causa (11).

El dolor musculoesquelético muchas veces es sub diagnosticado dando como resultado repercusiones negativas con un alto impacto social y económico: pruebas médicas innecesarias, fracaso en la mejoría de los síntomas, mayor tiempo para un diagnóstico correcto, frustración del paciente con afectaciones en su salud mental y una mayor carga en la prestación de asistencia sanitaria (12). Por tanto, un diagnóstico preciso es esencial para garantizar un manejo adecuado con óptimos resultados (4).

Presentamos y describimos el caso de una paciente remitida por una contractura muscular a nivel del trapecio superior (CIE-11). Al evaluarla aplicando la CIF en el ámbito clínico fisioterapéutico, es decir, ubicando las estructuras afectadas, definiendo las funciones alteradas, determinando las limitaciones en la actividad e identificando las restricciones en la participación, se encontró una disfunción generalizada en los músculos craneocervicomandibulares y de los miembros superiores, enmarcando entonces a la paciente en un síndrome de afectación miofascial con limitación en actividades de la vida diaria y afectación en la participación, situación que permitió un adecuado abordaje fisioterapéutico.

\section{CASO CLÍNICO}

Paciente de sexo femenino, de 52 ańos de edad, procedencia urbana y de ocupación docente universitaria, que asistió al servicio de fisioterapia de consulta externa en una entidad de I nivel de atención, con diagnóstico médico de contracturas musculares, refiriendo dolor en zona de trapecio superior y región cervical. Como antecedente importante, tuvo un síndrome de latigazo secundario a trauma a nivel cervical por accidente automovilístico en el ańo 2011, recibió manejo ortopédico con collar cervical por 6 meses, más antiinflamatorios no esteroideos (AINES) que prefirió no tomar.
El 6 de febrero de 2019 tuvo la valoración inicial por Fisioterapia previa firma del Consentimiento Informado; en la anamnesis refirió dolor en región cervical y miembros superiores, el dolor fue descrito como pulsante y extenuante en grado severo durante los últimos 7 días, calificado como insoportable según el cuestionario de dolor de McGill y de intensidad 10/10 según la Escala Visual Analógica del dolor (EVA).

En el examen físico, durante la exploración y palpación muscular se detectó la presencia de bandas tensas y Puntos Gatillos Miofasciales (PGM) a nivel de la región cervicodorsal y la musculatura general de los miembros superiores. La movilidad articular de columna cervical, hombros, codos, muńecas y manos se encontraba limitada por dolor y la evaluación funcional muscular denotaba una calificación de $\mathrm{R}$-(Movimiento incompleto contra gravedad sin resistencia) para músculos cervicales y de miembros superiores, generándole una limitación en las actividades de la vida diaria relacionadas con el cuidado personal y el vestido.

En la evaluación postural, se encontró un patrón postural espiratorio con disbalance en la esfera cervical y lumbar. En la palpación, se encontraron adherencias de la fascia a nivel toraco-lumbar, en músculos deltoides izquierdo y derecho, dolor en la zona nasociliar y zona de cigomáticos e hipersensibilidad en zona de cuero cabelludo.

Se aplicó el cuestionario SF-36 de calidad de vida, para explorar la salud física y la salud mental; escala de Barthel para detectar 10 actividades básicas de la vida diaria (ABVD); cuestionario DASH para determinar las discapacidades del hombro, codo y mano además de indagar acerca de sus síntomas y de su capacidad para llevar a cabo ciertas actividades; y cuestionario espańol del impacto de la fibromialgia para establecer un diagnóstico diferencial entre la presencia del síndrome miofascial y la presencia de fibromialgia (14). Ver Tabla 1.

Se indagaron factores contextuales y personales, la paciente se desempeńa como docente, labor que la obliga a estar varias horas sentada frente al computador; respecto a la actividad de dictar clase, expresó que no le genera mucha carga. Presentaba además variación en su estado de ánimo y dificultad para dormir en las noches. Prefiere no tomar ningún tipo de medicamentos porque no le "gustan" y refiere no tener estudios paraclínicos ni de imágenes diagnósticas.

De acuerdo con la Asociación Americana de Terapia Física (APTA), el estado de salud de la paciente se clasificó a nivel del patrón musculoesquelético en $\mathrm{D}$, lo que indica deficiencia en la movilidad articular, la función motora, el rendimiento 
Tabla 1. Escalas utilizadas para la valoración de la paciente utilizadas por los autores

\begin{tabular}{|c|c|c|}
\hline Escala & Resultados & Observaciones \\
\hline $\begin{array}{l}\text { Cuestionario de dolor } \\
\text { de McGuill }\end{array}$ & $\begin{array}{l}\text { Ultimos } 7 \text { días: El peor dolor de tipo extenuan- } \\
\text { te (severo). Actualmente insoportable }\end{array}$ & $\begin{array}{l}\text { La paciente refiere los puntajes más altos de cada ítem } \\
\text { en la escala }\end{array}$ \\
\hline \multirow{6}{*}{$\begin{array}{l}\text { Escala visual análoga } \\
\text { del dolor }\end{array}$} & 06/02/2019: 10/10 R. Escapulohumeral MSI & \multirow{6}{*}{$\begin{array}{l}\text { Se evidencia mejoría del dolor tras cada sesión de } \\
\text { fisioterapia }\end{array}$} \\
\hline & 08/02/2019: 10/10 R. Escapulohumeral MSI & \\
\hline & 11/02/2019: 7/10 R. Escapulohumeral MSI-MSD & \\
\hline & 13/02/2019: 7/10 R. Escapulohumeral MSI-MSD & \\
\hline & 15/02/2019: 6/10 R. Escapulohumeral MSI-MSD & \\
\hline & 27/02/2019: 6/10 R. escapulohumeral MSI-MSD & \\
\hline \multirow{8}{*}{$\begin{array}{l}\text { Custionario de Cali- } \\
\text { dad de vida SF-36 }\end{array}$} & Función física $60 \%$ & \multirow{8}{*}{$\begin{array}{l}\text { Se evidencia que la calidad de vida se ve afectada } \\
\text { principalmente por la presencia de dolor lo cual afect } \\
\text { su salud fisica y mental }\end{array}$} \\
\hline & Rol físico $25 \%$ & \\
\hline & Dolor Corporal 13\% & \\
\hline & Salud general $75 \%$ & \\
\hline & Vitalidad 50\% & \\
\hline & Función social $75 \%$ & \\
\hline & Rol emocional 33\% & \\
\hline & Salud mental $52 \%$ & \\
\hline Escala de Barthel & Dependencia Leve $>60$ pts & La paciente refiere necesitar ayuda para vestirse \\
\hline $\begin{array}{l}\text { Cuestionario espańol } \\
\text { de impacto de la } \\
\text { fbromialgia }\end{array}$ & $75 \mathrm{pts}$ & $\begin{array}{l}\text { El estado de salud y la capacidad funcional de la } \\
\text { paciente se encuentran muy afectados, se aplicó este } \\
\text { cuestionario para establecer un diagnóstico diferencial }\end{array}$ \\
\hline $\begin{array}{l}\text { Cuestionario DASH, } \\
\text { sobre las discapaci- } \\
\text { dades de la mano, } \\
\text { hombro y codo }\end{array}$ & $\begin{array}{l}\text { Puntuación de discapacidad/síntoma 24.1\% } \\
\text { Puntuación Trabajo/ocupación 18.7\% }\end{array}$ & $\begin{array}{l}\text { No muestra una discapacidad significativa según los } \\
\text { resultados de las escalas }\end{array}$ \\
\hline
\end{tabular}

muscular y los rangos de movimiento, asociados a disfunción del tejido conectivo. El patrón tegumentario comprometido fue el $\mathrm{B}$, presentando deficiencia de la integridad tegumentaria asociada con compromiso superficial de la piel.

A partir de los parámetros de la CIF, el diagnóstico fisioterapéutico de inicio fue: Deficiencia severa en estructuras relacionadas con el movimiento en la región cervicodorsal y músculos de los miembros superiores, deficiencia moderada en piel y estructuras relacionadas, deficiencia severa en las funciones sensoriales y dolor, deficiencias moderadas en funciones neuromusculoesqueléticas relacionadas con el movimiento de miembros superiores en todos sus rangos de movilidad y fuerza, y deficiencia severa en funciones mentales relacionadas con el sueńo y las emociones. Limitación moderada para mantener una posición por largos períodos de tiempo, limitación moderada en actividades de la vida diaria relacionadas con el cuidado personal y el vestido. Restricción en la participación para actividades de ocio y tiempo libre, como correr.

A partir de la evaluación fisioterapéutica, se establecieron como objetivos mejorar las deficiencias estructurales miofasciales, disminuir las deficiencias funcionales, mejorar la limitación en las actividades y reintegrar a la paciente a sus actividades de participación, estableciendo una mejora en la calidad de vida. En la tabla 2 se describen las técnicas empleadas en el plan de intervención terapéutica. 
Tabla 2. Tratamiento de modalidades físicas y técnicas terapéuticas establecidos por los autores

\begin{tabular}{|c|c|c|c|c|}
\hline Tipo & Grupo muscular & Articulación & $\begin{array}{l}\text { Duración/ } \\
\text { Repeticio- } \\
\text { nes }\end{array}$ & Efectos fisiológicos \\
\hline $\begin{array}{l}\text { Masaje } \\
\text { terapéutico } \\
\text { con técnica } \\
\text { de liberación } \\
\text { miofascial }\end{array}$ & $\begin{array}{l}\text { Cadena muscular } \\
\text { cervical profunda, } \\
\text { trapecio fibras } \\
\text { superiores y medias } \\
\text { bilateral, zona axilar } \\
\text { y pectorales }\end{array}$ & $\begin{array}{l}\text { Columna cervical, escapulo } \\
\text { torácica bilateral, acromiocla- } \\
\text { vicular bilateral, esternoclavi- } \\
\text { cular bilateral, atlantooccipi- } \\
\text { tal, reja costal }\end{array}$ & 3 minutos & $\begin{array}{l}\text { Aumento de la síntesis de colágeno, } \\
\text { condrocitos, elastina, citoquinas, y } \\
\text { factores de crecimiento, aumento del } \\
\text { aporte de oxígeno en el tejido. }\end{array}$ \\
\hline $\begin{array}{l}\text { Reflexología } \\
\text { del pie }\end{array}$ & $\begin{array}{l}\text { Región plantar } \\
\text { medial }\end{array}$ & & 10 minutos & $\begin{array}{l}\text { Promueve el flujo sanguíneo y linfático } \\
\text { y aumenta la flexibilidad de los mús- } \\
\text { culos basándose en la interconexión } \\
\text { existente entre los órganos internos y } \\
\text { las extremidades (zonas reflejas) rela- } \\
\text { cionadas entre sí, por medio del sistema } \\
\text { nervioso y los meridianos energéticos }\end{array}$ \\
\hline Masaje clásico & $\begin{array}{l}\text { Cara, cuero cabellu- } \\
\text { do, trapecio fibras } \\
\text { medias bilateral, } \\
\text { paravertebrales, } \\
\text { miembros superiores }\end{array}$ & $\begin{array}{l}\text { Columna cervical, dorsal y } \\
\text { lumbar, glenohumeral bilate- } \\
\text { ral, codo bilateral }\end{array}$ & 10 minutos & $\begin{array}{l}\text { Acción sedante, reducción de la } \\
\text { fatiga, debido al rápido vaciado de los } \\
\text { vasos de la zona tratada, intensifica } \\
\text { el flujo circulatorio, reduciendo el } \\
\text { estancamiento vascular, aumento en } \\
\text { temperatura local de piel. }\end{array}$ \\
\hline $\begin{array}{l}\text { Tracción cervi- } \\
\text { cal manual }\end{array}$ & Musculatura cervical & Columna cervical & 3 minutos & $\begin{array}{l}\text { Separa las superficies articulares, } \\
\text { reduce la p rotrusión de material } \\
\text { del nucleo discal, distiende las partes } \\
\text { blandas, relaja los músculos y moviliza } \\
\text { las articulaciones, reduciendo el dolor. }\end{array}$ \\
\hline $\begin{array}{l}\text { Ejercicios ac- } \\
\text { tivos resistidos }\end{array}$ & Miembros superiores & Glenohumeral bilateral & $\begin{array}{l}3 \text { series de } 5 \\
\text { repeticiones }\end{array}$ & $\begin{array}{l}\text { Aumento de la movilidad, eslasticidad } \\
\text { y resistencia }\end{array}$ \\
\hline $\begin{array}{l}\text { Termoterapia } \\
\text { superficial (ca- } \\
\text { lor húmedo) }\end{array}$ & $\begin{array}{l}\text { Musculatura cervical } \\
\text { y miembros supe- } \\
\text { riores }\end{array}$ & $\begin{array}{l}\text { Zona de aplicación: región } \\
\text { cervico-dorsal y braquial } \\
\text { izquierdo }\end{array}$ & 20 minutos & $\begin{array}{l}\text { Efectos hemodinámicos: Vasodilata- } \\
\text { ción, aumento de temperatura en zona, } \\
\text { aumento de liberación de vasodilatado- } \\
\text { res, relajación muscular, disminución } \\
\text { de la actividad simpática. Efectos } \\
\text { Neuromusculares: Cambios en la } \\
\text { velodad de conducción nerviosa y en } \\
\text { la frecuencia de descarga, aumento del } \\
\text { umbral del dolor, cambios en la fuerza } \\
\text { muscular. } \\
\text { Metabólicos: Aumento de la velo- } \\
\text { cidad de las reacciones biológicas } \\
\text { enzimáticas, aumento de los arcos de } \\
\text { movilidad y disminución de la rigidez } \\
\text { articular. }\end{array}$ \\
\hline $\begin{array}{l}\text { Ultrasoni- } \\
\text { do: modo } \\
\text { continuo: Fre- } \\
\text { cuencia } 1 \mathrm{Mhz}, \\
\text { Intensidad } 2,4 \\
\mathrm{~W} / \mathrm{cm} 2\end{array}$ & $\begin{array}{l}\text { Musculatura cervical } \\
\text { y miembros supe- } \\
\text { riores }\end{array}$ & $\begin{array}{l}\text { Zona de aplicación: región del } \\
\text { braquial MSI y del trapecio } \\
\text { fibras superiores y medias } \\
\text { bilateral acompańando la } \\
\text { aplicación con estiramiento }\end{array}$ & 5 minutos & $\begin{array}{l}\text { Aceleración del metabolismo, reduc- } \\
\text { ción del dolor y del espasmo muscular, } \\
\text { aceleración de la velocidad de conduc- } \\
\text { ción nerviosa, aumento del flujo de } \\
\text { sangre, aumento de la extensibilidad } \\
\text { de los tejidos. }\end{array}$ \\
\hline
\end{tabular}


La intervención fisioterapéutica fue programada con 4 sesiones semanales de 60 minutos de duración hasta completar las 15 sesiones, cumpliendo con la presencialidad del fisioterapeuta. En las primeras cinco sesiones se realizaron técnicas manuales de masaje clásico y liberación miofascial superficial en zona cervical, uso de técnica de manos cruzadas en la zona pectoral mayor y menor, liberación miofascial superficial en miembros superiores, aplicación de modalidades físicas de calor húmedo en cuello, ultrasonido en región cervicodorsal y hombro izquierdo.

En las siguientes cinco sesiones se disminuyó la frecuencia, intensidad y duración de aplicación de las técnicas manuales y de las modalidades físicas, implementando ejercicios funcionales generales como trabajo en polea, escalerilla para dedos, máquina de contrapesos, pelotas y bandas elásticas a fin de mejorar la resistencia al ejercicio, fortalecer la musculatura involucrada y mejorar la capacidad funcional. El tratamiento fisioterapéutico incluyó también reflexología en zona plantar medial bilateral y otras técnicas como tracción cervical y estiramientos miofasciales combinados con la aplicación de ultrasonido.
En las últimas sesiones se hizo énfasis en el trabajo funcional muscular y se finalizó con una técnica de relajación para ayudar a modular los estados de ánimo negativos, además se realizó educación a la paciente mediante un plan casero respecto a la higiene postural y estiramientos de las cadenas miofasciales para el manejo del dolor en las actividades de la vida diaria.

El seguimiento del umbral del dolor se realizó a través de la EVA, efectuando 7 mediciones desde la fecha inicial de evaluación hasta la última sesión de fisioterapia, la movilidad articular se realizó de manera pasiva, la evaluación muscular se realizó con la escala de evaluación funcional muscular, las limitaciones y restricciones se evaluaron con el trabajo de ejercicios activos y la restricción de la participación a partir de la información dada por la paciente.

Al finalizar el abordaje fisioterapéutico hubo una notable disminución del dolor pasando de 10/10 a 4/10 según la EVA, lo cual influyó positivamente en la mejoría de las condiciones estructurales del sistema musculo-esquelético, como la postura, la funcionalidad y la realización de

Tabla 3. Evolución cronológica del estado de salud de la paciente.

\begin{tabular}{|c|c|c|c|c|c|}
\hline $\begin{array}{l}\text { Fecha/ } \\
\text { sesión }\end{array}$ & $\begin{array}{l}\text { Dolor } \\
\text { EVA }\end{array}$ & $\begin{array}{l}\text { Movilidad } \\
\text { Articular }\end{array}$ & $\begin{array}{l}\text { Evaluación } \\
\text { funcional } \\
\text { muscular }\end{array}$ & $\begin{array}{l}\text { Limitación en Activi- } \\
\text { dad }\end{array}$ & Restricción en la participación \\
\hline $\begin{array}{l}6 / 02 / 2019 / 1 \\
\text { sesión }\end{array}$ & $10 / 10$ & $\begin{array}{l}\text { Limitada por } \\
\text { dolor }\end{array}$ & R- & $\begin{array}{l}\text { Limitación Moderada } \\
\text { en actividades Cuidado } \\
\text { personal y vestido }\end{array}$ & $\begin{array}{l}\text { Problema Grave Para actividades de } \\
\text { ocio y tiempo libre }\end{array}$ \\
\hline $\begin{array}{l}8 / 02 / 2019 / 2 \\
\text { sesión }\end{array}$ & $10 / 10$ & $\begin{array}{l}\text { Limitada por } \\
\text { dolor }\end{array}$ & R- & $\begin{array}{l}\text { Limitación Moderada } \\
\text { en actividades Cuidado } \\
\text { personal y vestido }\end{array}$ & $\begin{array}{l}\text { Problema grave para actividades de } \\
\text { ocio y tiempo libre }\end{array}$ \\
\hline $\begin{array}{l}11 / 02 / 2019 / 3 \\
\text { sesión }\end{array}$ & $7 / 10$ & $\begin{array}{l}\text { Limitación } \\
\text { moderada }\end{array}$ & $\mathrm{R}$ & $\begin{array}{l}\text { Limitación leve en activi- } \\
\text { dades Cuidado personal } \\
\text { y vestido }\end{array}$ & $\begin{array}{l}\text { Problema moderado para actividades } \\
\text { de ocio y tiempo libre }\end{array}$ \\
\hline $\begin{array}{l}13 / 02 / 2019 / 5 \\
\text { sesión }\end{array}$ & $7 / 10$ & $\begin{array}{l}\text { Limitación } \\
\text { leve }\end{array}$ & $\mathrm{R}$ & $\begin{array}{l}\text { Limitación leve para } \\
\text { actividades de cuidado } \\
\text { personal y vestido }\end{array}$ & $\begin{array}{l}\text { Problema moderado para actividades } \\
\text { de ocio y tiempo libre }\end{array}$ \\
\hline $\begin{array}{l}15 / 02 / 2019 / 6 \\
\text { sesión }\end{array}$ & $6 / 10$ & $\begin{array}{l}\text { Limitación } \\
\text { leve }\end{array}$ & $\mathrm{R}$ & Sin limitación & $\begin{array}{l}\text { Problema moderado para actividades } \\
\text { de ocio y tiempo libre }\end{array}$ \\
\hline $\begin{array}{l}20 / 02 / 2019 / \\
9 \text { sesión }\end{array}$ & $5 / 10$ & Sin limitación & $\mathrm{R}^{+}$ & Sin limitación & $\begin{array}{l}\text { Problema leve para actividades de } \\
\text { ocio y tiempo libre }\end{array}$ \\
\hline $\begin{array}{l}28 / 02 / 2019 / \\
15 \text { sesión }\end{array}$ & $4 / 10$ & Sin limitación & $\mathrm{R}^{+}$ & Sin Limitación & $\begin{array}{l}\text { Sin Problema para actividades de ocio } \\
\text { y tiempo libre }\end{array}$ \\
\hline
\end{tabular}

Fuente: Elaboración propia. 
actividades de la vida relacionadas con el cuidado personal y el vestido, mejorando su nivel de independencia. Además, la paciente refirió y mostró un mejor aspecto general, tanto físico como emocional, con retorno a sus actividades de ocio y esparcimiento. En la Tabla 3 se reporta la Evolución cronológica del estado de salud de la paciente. Asociado, se realizó remisión a Psiquiatría por el antecedente del trastorno del sueńo y emocional.

El diagnóstico fisioterapéutico de egreso fue: Deficiencia leve en estructuras relacionadas con el movimiento en la región cervicodorsal y músculos de los miembros superiores, sin deficiencia en piel y estructuras relacionadas, deficiencia leve en las funciones sensoriales y dolor, sin deficiencia en funciones neuromusculoesqueléticas y relacionadas con el movimiento de miembros superiores en todos sus rangos de movilidad y fuerza y deficiencia leve en funciones mentales relacionadas con el sueńo y las emociones.

La paciente evolucionó sin limitación para mantener una posición por largos periodos de tiempo, sin limitación en actividades de la vida diaria relacionadas con el cuidado personal y el vestido. Sin restricción en la participación para actividades de ocio, tiempo libre y ejercicio, como correr. En el manejo interdisciplinario, el médico general autorizó nuevas sesiones de fisioterapia y entregó remisión para el servicio de Psiquiatría.

\section{DISCUSIÓN}

En lo pertinente al caso, los profesionales de la salud tratantes realizaron un diagnóstico ampliado y correcto, apoyados en el uso de la CIF que les permitió diseńar un plan terapéutico adecuado y exitoso en la paciente. La información sobre el diagnóstico unida a la del funcionamiento, proporciona una visión global del estado de salud de las personas a partir de los componentes de la salud, propendiendo por devolver a los pacientes la calidad de vida que se pierde a causa de la falta de movilidad.

Además, para mayor comprensión del desarrollo de la patología de la paciente, se exploró el estado de salud por niveles según el planteamiento de Wang en donde la composición corporal implica el estudio de componentes que se organizan en cinco niveles de complejidad creciente: atómico, molecular, celular, sistema tisular y orgánico $(20,21)$. Las estructuras de la paciente comprometidas en sus diferentes niveles se observan en la Tabla 4, precisamente, cualquier cambio importante en la composición corporal se manifestará en todo el cuerpo (22).
El plan de intervención propuesto por los autores, se basó en los beneficios de la terapia manual a nivel periférico (mejora de la movilidad y disminución de la tensión muscular) y a nivel central (efecto analgésico), puesto que la movilización de las articulaciones produce una activación de las vías descendentes antinociceptivas y la terapia manual aplicada a nivel cervical denota ser efectiva en la reducción de la hiperexcitabilidad sensorial en pacientes con dolor crónico (14-17).

Para las fases agudas de dolor, aunque no hay evidencias disponibles que apoyen la efectividad de tratamientos exclusivos de fisioterapia, el mantenimiento del ejercicio es ampliamente recomendado (18). Se usaron técnicas cognitivas-conductuales sumadas a un programa progresivo de ejercicio, apoyado en la educación del paciente, las cuales reducen la intensidad del dolor y mejoran la función motora (19). Además, estímulos térmicos con aplicación de calor superficial y profundo, que en pacientes con sensibilización central al dolor han mostrado disminución de los umbrales del dolor (17).

El abordaje de Fisioterapia estuvo inmerso en el modelo de práctica clínica propuesto por la Asociación Americana de Terapia Física (APTA) en consonancia con la CIF, para dar un manejo asertivo, a partir de la identificación de las deficiencias en estructuras, funciones, limitaciones en la actividad y restricción en la participación, y a partir de allí generar un diagnóstico fisioterapéutico, un plan de intervención y un pronóstico funcional con el cual se encontrarán resultados positivos en un corto periodo de tiempo y que perdurarán más allá de la interacción y garantizarán un mejor pronóstico funcional, involucrando activamente al usuario en el proceso de recuperación para lograr una mayor adherencia al tratamiento.

En relación a lo anteriormente mencionado, se sugiere que el tratamiento fisioterapéutico orientado hacia la disminución y/o modulación del dolor desde su origen, a través de mecanismos directos e indirectos sobre las estructuras comprometidas en sus diferentes niveles, pueden ser efectivos para el abordaje del síndrome de dolor miofascial evidenciándose aumento de la movilidad articular y por ende la funcionalidad (6). El tratamiento propuesto se manejó desde la génesis patológica, la funcionalidad, la educación postural, la educación en técnicas de afrontamiento para el trabajo de las emociones y trabajando en la paciente la motivación a continuar con las actividades de ocio, esparcimiento y ejercicio como parte fundamental de la salud mental. Existe evidencia de los beneficios psicológicos del ejercicio (23), implicando un abordaje integral. 
Tabla 4. Estado de Salud por niveles del síndrome miofascial. Propuesta elaborada por los autores

\begin{tabular}{|c|c|c|c|c|c|}
\hline $\begin{array}{l}\text { Estructura } \\
\text { afectada }\end{array}$ & Atómico & Molecular & Celular & Histico & Orgánico \\
\hline MÚSCULO & $\begin{array}{l}\text { Déficit de } \\
\text { carbono, hidró- } \\
\text { geno, oxígeno, } \\
\text { calcio, magne- } \\
\text { sio, potasio. }\end{array}$ & $\begin{array}{l}\text { Alteración en el agua, } \\
\text { alteración en el meta- } \\
\text { bolismo de la actina, } \\
\text { miosina, carbohidra- } \\
\text { tos, proteínas y lípidos }\end{array}$ & $\begin{array}{l}\text { Alteración en la } \\
\text { función del retículo } \\
\text { endoplasmático } \\
\text { y en la función } \\
\text { mitocondrial del } \\
\text { miocito afectando la } \\
\text { producción de ATP }\end{array}$ & $\begin{array}{l}\text { Deficiencia a nivel de: } \\
\text { S. Musculoesquelético } \\
\text { S. Nervioso (límbico) } \\
\text { S. Cardiovascular } \\
\text { S. Linfático } \\
\text { S. tegumentario }\end{array}$ & $\begin{array}{l}\text { Deficiencia a nivel de: } \\
\text { Músculos longuísimos } \\
\text { del cuello y cabeza, } \\
\text { trapecio superior y } \\
\text { medio, romboideos, } \\
\text { deltoides, muscula- } \\
\text { tura general del m. } \\
\text { superiores. }\end{array}$ \\
\hline LIGAMENTO & $\begin{array}{l}\text { Déficit de } \\
\text { carbono, } \\
\text { hidrógeno, } \\
\text { oxígeno, azufre, } \\
\text { nitrógeno }\end{array}$ & $\begin{array}{l}\text { Alteración en el agua, } \\
\text { aminoácidos coláge- } \\
\text { nos, y elastina }\end{array}$ & $\begin{array}{l}\text { Alteración en la fun- } \\
\text { ción del fibroblasto } \\
\text { por desequilibrio } \\
\text { en la producción de } \\
\text { colágeno y elastina } \\
\text { y ensamble de estos } \\
\text { por acción de los } \\
\text { ribosomas }\end{array}$ & $\begin{array}{l}\text { Deficiencia a nivel de: } \\
\text { S. Musculoesquelético } \\
\text { S. Nervioso } \\
\text { S. Cardiovascular }\end{array}$ & $\begin{array}{l}\text { Deficiencia a nivel de } \\
\text { ligamentos de las arti- } \\
\text { culaciones cervicales, } \\
\text { escapulohumerales, } \\
\text { escapulotorácicas y } \\
\text { glenohumerales }\end{array}$ \\
\hline ARTICULACIÓN & $\begin{array}{l}\text { Déficit de } \\
\text { carbono, } \\
\text { hidrógeno, } \\
\text { oxígeno, azufre, } \\
\text { nitrógeno }\end{array}$ & $\begin{array}{l}\text { Alteración en el agua, } \\
\text { alteración en el meta- } \\
\text { bolismo del colágeno, } \\
\text { proteínas, glucosami- } \\
\text { noglicanos }\end{array}$ & $\begin{array}{l}\text { Alteración en la } \\
\text { función de los con- } \\
\text { drocitos al formar y } \\
\text { mantener la matriz } \\
\text { extracelular }\end{array}$ & $\begin{array}{l}\text { Deficiencia a nivel de: } \\
\text { S. Musculoesquelético } \\
\text { S. Nervioso (propio- } \\
\text { ceptivo) } \\
\text { S. Cardiovascular }\end{array}$ & $\begin{array}{l}\text { Deficiencia a nivel de } \\
\text { ligamentos de las arti- } \\
\text { culaciones cervicales, } \\
\text { escapulohumerales, } \\
\text { escapulotorácicas y } \\
\text { glenohumerales }\end{array}$ \\
\hline
\end{tabular}

Como limitación encontramos que el desconocimiento de otro tipo de clasificaciones del estado de salud pudo generar la demora de la atención integral de la paciente, disminuyendo su funcionalidad y calidad de vida por varios ańos.

\section{CONCLUSIÓN}

Este caso clínico da a conocer varios aspectos importantes en cuanto a la génesis de la patología en mención, el diseńo de un plan terapéutico teniendo en cuenta la importancia de valorar el estado de salud de una persona de una forma integral utilizando otro tipo de clasificación, la clasificación internacional del funcionamiento, de la discapacidad y de la salud. Este caso aporta información acerca de los beneficios que se pueden obtener con las diferentes estrategias terapéuticas convencionales que ofrece la fisioterapia y permite orientar las acciones del abordaje clínico-terapéutico desde una mirada biopsicosocial.

\section{CONTRIBUCIÓN DE LOS AUTORES}

Todos los autores declaran contribución en todas las fases de desarrollo de este manuscrito.

\section{AGRADECIMIENTOS}

Lo que dicen en el parrafo.

\section{DECLARACIÓN DE CONFLICTO DE INTERESES}

Los autores declaran no tener ningún conflicto de intereses en la realización de este estudio.

\section{FINANCIACIÓN}

Ninguna. 


\section{REFERENCIAS}

1. Mezzich JE, Zapata MI, Schwartz K. La nueva clasificación internacional de enfermedades y el diagnóstico multiaxial en medicina general. Rev Med Hered . 1995; 6(4):187-192.

2. Fernández JA, Fernández-Fidalgo M, Alarcos C. Los conceptos de calidad de vida, salud y bienestar analizados desde la perspectiva de la Clasificación Internacional del Funcionamiento (CIF). Rev. Esp. Salud Publica. 2010; 84(2):169-184.

3. Organización Mundial de la Salud. Clasificación Internacional del funcionamiento, de la discapacidad y de la salud CIF. ISBN 924154544-5, 2001. Disponible en: https://apps.who.int/iris/bitstream/ handle/10665/43360/9241545445_spa.pdf?sequence=1

4. Hernán-Garcia M, Garcia D, Cubillo J, Cofino R. Fundamentos del enfoque de activos para la salud en atención primaria de salud. FMC. 2019; 26 (1):1-9. DOI: 10.1016/j.fmc.2019.06.005

5. Bourgaize S, Newton G, Kumbhare D, Srbely J. A comparison of the clinical manifestation and pathophysiology of myofascial pain syndrome and fibromyalgia: implications for differential diagnosis and management. The Journal of the Canadian Chiropractic Association. 2018; 62(1):26-41.

6. Laimi K, Mäkilä A, Bärlund E, Katajapuu N, Oksanen A, Seikkula V, Saltychev M. Effectiveness of myofascial release in treatment of chronic musculoskeletal pain: a systematic review. Clinical Rehabilitation. 2018; 32(4):440-450. DOI. 10.1177/0269215517732820

7. Cabaynes Truffino J. Protocolo diagnóstico de las contracturas musculares. Medicine. 2015; 11(75):45284531. DOI: 10.1016/j.med.2015.03.005

8. Daza JS, Iglesias A. Dolor Musculoesquelético. ACED, Editora Guadalupe S.A. 671p, 2010.

9. Saxena A, Chansoria M, Tomar G, Kumar A. Myofascial pain syndrome: an overview. Journal of Pain \& Palliative Care Pharmacotherapy. 2015; 29(1):16-21. DOI: https:// doi.org/10.3109/15360288.2014.997853

10. Arranz-Martín B, Navarro-Brazález B. Abordaje fisioterapéutico del síndrome de dolor miofascial tras cirugía reconstructiva por cáncer de mama: estudio de caso. Fisioterapia. 2018 ;40(5):273-277. DOI: https://doi. org/10.1016/j.ft.2018.05.001

11. Serrano-Muńoz D, Gomez-Soriano J, Avila-Martín G, Galán-Arriero I, Romero-Muńoz LM, Taylor J, et al. Sensibilización central al dolor en pacientes con síndrome del latigazo cervical: Una revisión. Revista Latinoamérica de Cirugía Ortopédica. 2016; 1(3):102107. DOI: 10.1016/j.rslaot.2017.02.001
12. Velasco M. Dolor musculoesquelético: Fibromialgia y síndrome de dolor miofacial. Revista Médica Clínica las Condes. 2019; 30(6):414-427.

13. Van Oosterwijck J, Nijs J, Meeus M, Paul L. Evidence for central sensitization in chronic whiplash: A systematic literature review. Eur J Pain. 2013; 17(3):299-312. DOI: 10.1002/j.1532-2149.2012.00193.x

14. Chavarría J. Síndrome de dolor miofascial, diagnóstico y tratamiento. Revista Médica de Costa Rica y Centroamérica LXXI. 2014; (612): 683-689.

15. Pooja S, Pradeep S. Advances in the management of myofascial pain dysfunction syndrome - A short review. Drug Invention Today. 2019; 12(2):309-13.

16. Bourgaize S, Srbely J, Newton G, Janjua I, Murnaghan K, Mior S. Fibromyalgia and myofascial pain syndrome: Two sides of the same coin? A scoping review to determine the lexicon of the current diagnostic criteria. Musculoskeletal Care. 2019; 17(1): 3-12. DOI: 10.1002/msc.1366

17. Nijs J, Malfliet A, Ickmans K, Baert I, Meeus M. Treatment of central sensitization in patients with 'unexplained' chronic pain: An update. Expert Opin Pharmacother. 2014; 15:1671-1683. DOI: 10.1517/14656566.2014.925446

18. Peolsson A, Landen-Ludvigsson M, Overmeer T, Dedering A, Bernfort L, Johansson G, et al. Effects of neck-specific exercise with or without a behavioural approach in addition to prescribed physical activity for individuals with chronic whiplash-associated disorders: A prospective randomised study. BMC Musculoskelet Disord. 2013; 14:311. DOI: 10.1186/1471-2474-14-311

19. Stewart MJ, Maher CG, Refshauge KM, Herbert RD, Bogduk N, Nicholas M.

20. Randomized controlled trial of exercise for chronic whiplash-associated disorders. Pain. 2007; 128: 59-68. DOI: 10.1016/j.pain.2006.08.030

21. Wang ZM, Heshka S, Pierson RN, Heymsfield SB. Systematic organization of body-composition methodology: an overview with emphasis on component-based methods. The American Journal of Clinical Nutrition. 1995; 61(3): 457-465. D0I: 10.1093/ ajcn/61.3.457

22. Ledezma FP, Vernaza-Pinzón P. Enfermedad de Kienböck: Reporte de un caso. Revista facultad Ciencias de la Salud. Universidad del Cauca. 2013; 15(3): 41-45.

23. Wang ZM, Pierson RN, Heymsfield SB. The five-level model: a new approach to organizing body-composition research. The American Journal of Clinical Nutrition. 1992; 56(1):19-28. DOI: 10.1093/ajcn/56.1.19

24. Kaur J, Garnawat D. The mental health benefits of physiotherapy. Fysioterapeuten. 2016; 7: 33-36. 